\title{
A young woman with myalgia
}

\author{
Authors: Abarna Ratnarajah, ${ }^{A}$ Brendan Barber, ${ }^{B}$ Yohan Samarasinghe ${ }^{C}$ and Mark Lloyd ${ }^{D}$
}

\begin{abstract}
We describe the case of a young female presenting with myalgia, mildly raised creatine kinase and a rash. The discussion emphasises the importance of a systematic approach to muscle symptoms, the value of a detailed hand examination and the importance of magnetic resonance imaging in the investigation of muscular symptoms.
\end{abstract}

KEYWORDS: Muscle, myalgia, rash

\section{Case presentation}

A 37-year-old female chef was referred by her general practitioner (GP) with 1 week's history of proximal upper limb myalgia with subjective weakness, which worsened throughout the day, and a 5-day history of breathlessness. She described the aching as being like a 'lactic acid build up'. She denied chest pain, fever, haemoptysis, cough or recent travel. She had $20 \mathrm{~kg}$ intentional weight loss. Her past medical history was significant for type 2 diabetes, polycystic ovarian syndrome and carpal tunnel syndrome. She had also recently been found to be vitamin D deficient (normal serum calcium and alkaline phosphatase). Her medications included metformin and overthe-counter vitamin D. She was teetotal and did not smoke. There was no history of illicit drug use and no family history of neuromuscular disease.

On admission, the patient was afebrile and haemodynamically stable, with a blood pressure of $136 / 85 \mathrm{mmHg}$ and heart rate of 56 beats per minute. $\mathrm{Her}_{2}$ saturations were $100 \%$ on air and she had a respiratory rate of 16 . Her cardiovascular, respiratory and abdominal system examinations were unremarkable. Her cranial nerves were intact and her peripheral nervous system examination was also normal. Specifically, no objective weakness or reduced sensation was elicited and reflexes were intact.

Normal or negative initial investigations included full blood count, urea and electrolytes, calcium, liver and thyroid function tests, fasting lactate, C-reactive protein, chest X-ray and electrocardiogram (ECG), arterial blood gases and cardiac troponin.

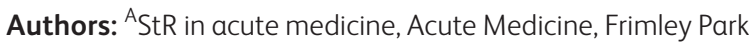
Hospital, Frimley, UK; ${ }^{\text {B }}$ consultant in radiology, Frimley Park Hospital, Frimley, UK; ${ }^{C}$ consultant in acute medicine, Frimley Park Hospital, Frimley, UK; ${ }^{D}$ consultant in rheumatology, Frimley Park Hospital, Frimley, UK

\section{What is the differential diagnosis?}

This patient presented with proximal symmetrical myalgia with subjective weakness and an element of proximal lower limb fatiguability. The fatiguability and symmetrical symptoms led to myasthenia gravis being considered, but there was no evidence of ptosis, bulbar symptoms or reflex loss. ${ }^{1}$

Hyperthyroidism and more commonly hypothyroidism can both present with proximal myopathy. Patients with hyperthyroidism may also complain of weight loss, diarrhoea and heat intolerance. ${ }^{1}$ Those with hypothyroidism commonly have an elevated creatinine kinase (CK).

Cushing's syndrome (endogenous and exogenous) is another differential for proximal myopathy. ${ }^{1}$ Patients with Cushing's syndrome may have weight gain, striae, thinning of the skin, hirsuitism, acne and, in women, oligo- or amenorrhoea. ${ }^{1}$

Alcohol excess can also cause proximal muscle weakness, but the patient was teetotal. ${ }^{1}$

Viral myositis, for instance secondary to human immunodeficiency virus (HIV) or influenza, is also a possible differential.

As no objective weakness was elicited, polymyalgia rheumatica was considered. ${ }^{2}$ This can cause symptoms that are suggestive of proximal myalgia but it rarely affects those under the age of 50 years. ${ }^{2}$ The symptoms are caused by referred pain from shoulders and hips; the muscles are not affected. ${ }^{2}$ Inflammatory markers are usually raised.

Metabolic myopathies, classically muscle phosphorylase deficiency (McArdle disease), can present with weakness and poor exercise tolerance in adult life, but the history is usually chronic rather than acute. ${ }^{2}$

Finally, inflammatory myopathies, including dermatomyositis (DM), polymyositis (PM) and myositis associated with other autoimmune conditions, also produce proximal muscle weakness. ${ }^{1}$ The peak incidence of DM and PM is between 40 and 50 years of age. ${ }^{3}$

\section{Case progression}

On the post-take ward round, clinicians felt that this woman had fatiguability and she also stated that her speech could be affected. A neurology opinion was requested to look at the possibility of myasthenia gravis. Concurrently, some bloods returned. The patient's thyroid function test was normal, her D-dimer was positive at $607 \mu \mathrm{g} / \mathrm{l}$ (normal range $<500 \mu \mathrm{g} / \mathrm{l}$ ) and her creatine kinase (CK) was mildly raised at $490 \mathrm{IU} / 1$ (normal range 25-200 IU/l). As she was breathless, with a positive D-dimer, a computed tomography pulmonary angiogram (CTPA) 


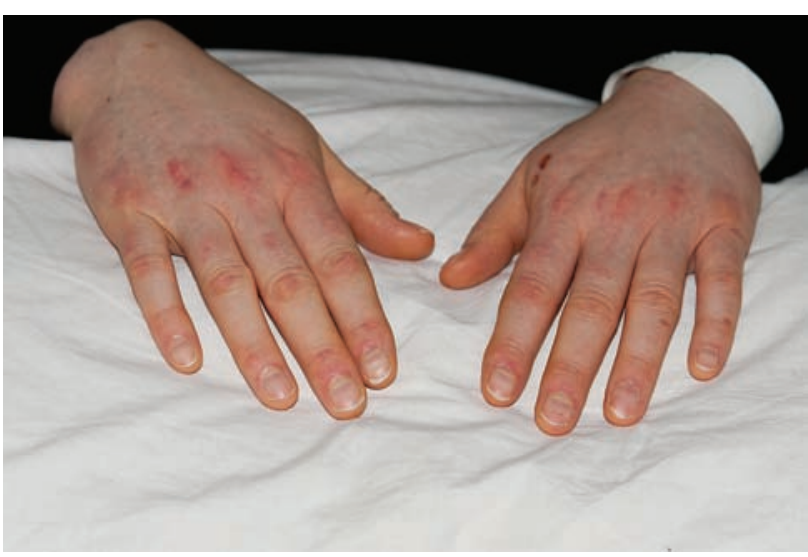

Fig 1. Gottrons papules: erythematous papules overlying the metacarpal and interphalangeal joints.

was performed, which excluded a pulmonary embolus. Myositis-associated antibodies were also measured.

Neurology review revealed no ptosis, swallowing or speech problems. It was concluded that there was limited evidence to support myasthenia gravis. A fasting lactate was normal, as was the patient's forced vital capacity (FVC) was normal at 3.291 (predicted 3.27 1). She was, however, noted to have a non-blanching rash on her hands (Fig 1). This had been present for several weeks and was previously diagnosed as a reactive dermatitis. The rheumatology team reviewed the patient and noted dilated nail fold capillaries and long cuticles with tiny infarcts (Fig 2). The knuckle rash was felt to be consistent with Gottrons papules. The patient also demonstrated fatiguablity on stands test, managing only six stands from a sitting position without using her arms. She also became breathless during the test, though she did not desaturate.

A diagnosis of dermatomyositis was made on clinical grounds. An urgent magnetic resonance imaging (MRI) scan of her shoulders showed extensive oedema throughout all muscle groups, consistent with myositis (Fig 3). Anti-nuclear antibody was positive at titre $1 / 320$. Further immunology, including anti Jo-1 and extended myositis panel, was negative. Treatment

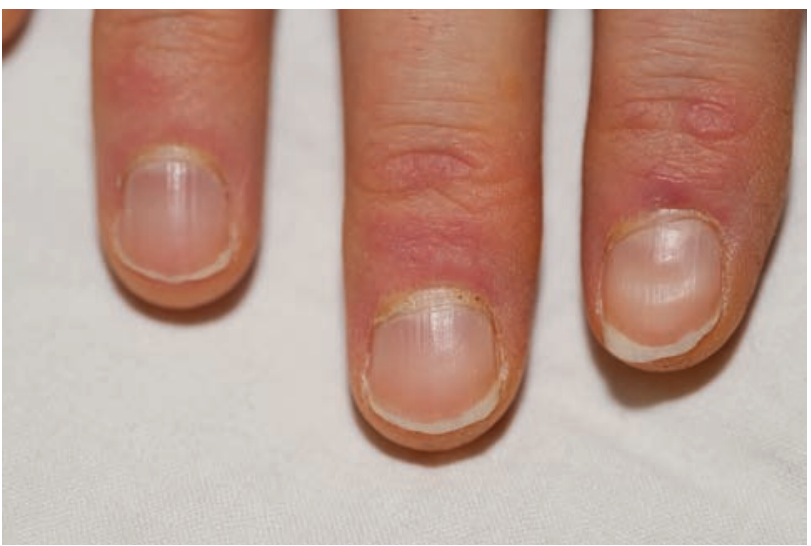

Fig 2. Dilated nail fold capillaries and long cuticles with tiny infarcts.

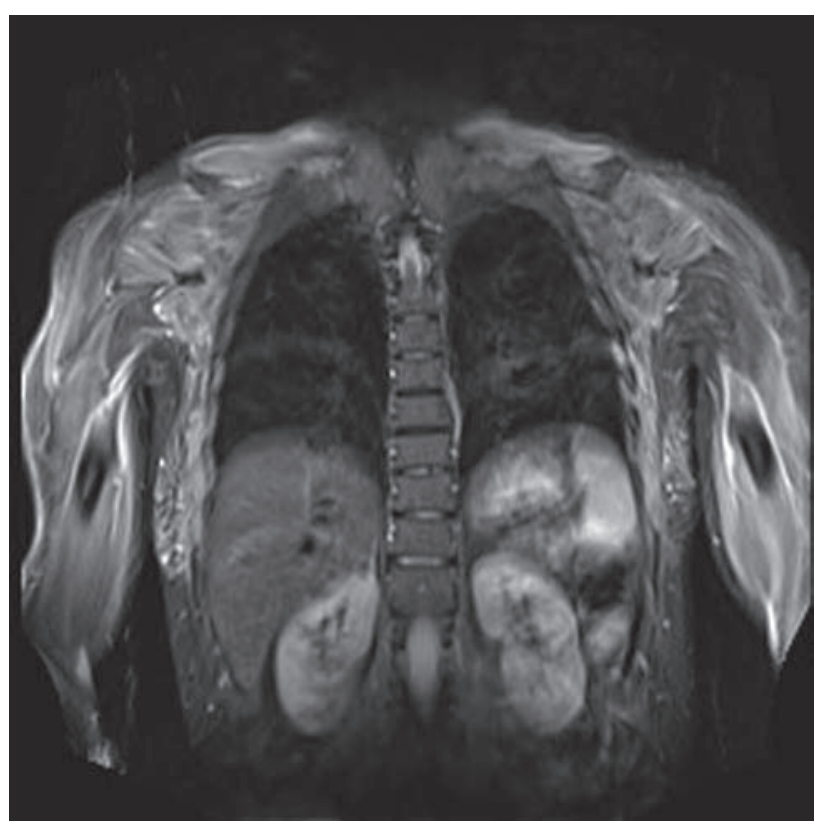

Fig 3. Magnetic resonance imaging (MRI) of the shoulders and thorax with coronal short tau inversion recovery (STIR) sequence taken before steroid therapy. The image shows diffuse hyperintense signal within the muscles of the upper limb and shoulder girdle, consistent with oedema.

with prednisolone $30 \mathrm{mg}$ daily was started, with a rapid but not complete fall in CK levels. These levels improved to normal (43 IU/1) with resolution of MRI changes (Fig 4) following the introduction of methotrexate $15 \mathrm{mg}$ weekly and a temporary increase in prednisolone to $60 \mathrm{mg}$ daily. Electromyography (EMG) 2 weeks after the start of treatment was normal. Because of a family history of breast cancer, the patient had a mammogram and pelvic ultrasound, both of which were normal.

\section{Discussion}

Dermatomyositis is one of the idiopathic inflammatory myopathies; the others being polymyositis, immune-mediated necrotising myopathy and inclusion body myositis. ${ }^{3}$ It is more common in females than in males (2:1) with a peak incidence between the ages of 40 and 50 years, though it can affect any age group. ${ }^{4}$

Skin manifestations include a heliotrope rash (a purplish discolouration of the eyelids) and Gottrons papules, a violaceous rash that is prominent over the metacarpophalangeal or interphalangeal joints. ${ }^{1}$ This rash is pathognomonic for dermatomyositis and may precede or accompany the weakness. ${ }^{3}$ In contrast to the rash seen in systemic lupus erythematosus (SLE), this rash spares the phalanges. ${ }^{4}$ Other skin manifestations may include the shawl sign, an erythematous macular sun sensitive rash on the upper back, or the V sign, a rash on the anterior chest, face and neck. ${ }^{4}$ More subtle hand signs include periungual telangiectasiae and hypertrophy of the cuticles. A sub group of patients, those with amyopathic dermatomyositis, present with skin features alone without weakness or elevated enzymes. ${ }^{4}$ 


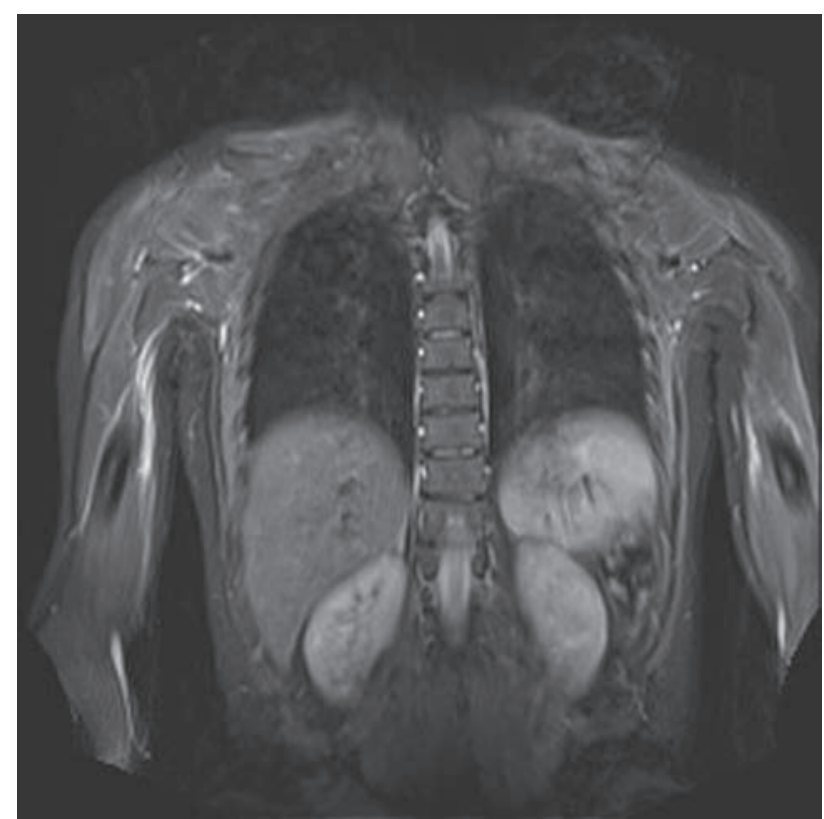

Fig 4. Magnetic resonance imaging (MRI) of the shoulders and thorax with coronal short tau inversion recovery (STIR) sequence taken after steroid therapy. The image shows a marked reduction of the oedema within the muscles compared to Fig 3.

Dermatomyositis classically affects the proximal skeletal muscles. ${ }^{4}$ Patients may describe difficulty standing from a squatting position or climbing stairs. ${ }^{4}$ It is crucial to document FVC to assess the degree of respiratory muscle involvement. In our case, we felt that the patient's dyspnoea was probably secondary to increased aerobic effort secondary to her reduced functional muscle capacity. Other systems can be affected, including the joints, oesophagus and lungs. ${ }^{1}$ Patients may also complain of arthritis, dysphagia and dyspnoea or cough secondary to interstitial lung disease. ${ }^{1}$ Cardiomyopathy, though a less-frequent complication, may present with arrhythmias or congestive cardiac failure. ${ }^{4}$ Cardiac troponin should therefore be checked in all patients. In severe cases, patients may present with pseudohaematuria resulting from myoglobulinaemia. ${ }^{2}$

Initial investigations include tests for elevated muscle enzymes. The most sensitive test is that for CK; other tests that may indicate muscle necrosis include those for lactate dehydrogenase (LDH), aldolase, aspartate aminotransferase (AST) and alanine aminotransferase (ALT).${ }^{1} \mathrm{CK}$ is elevated in $70 \%$ of cases, but CK levels do not correlate to weakness and may be normal. ${ }^{3}$ In our case, the patient's CK level initially appeared to be only mildly elevated, but based on subsequent levels, it was eventually raised tenfold. Myositis-specific antibodies can be helpful in categorising autoimmune inflammatory myopathy syndromes. For instance, the presence of anti-synthetase antibodies, such as anti-Jo 1 antibodies, may be associated with interstitial lung disease and Raynaud phenomenon. ${ }^{3}$ Muscle electrophysiology is not a diagnostic test but fibrillations and low-amplitude short-duration polyphasic potential may support the diagnosis. Muscle biopsy may show an inflammatory cell infiltrate, necrosis and phagocytosis of

\section{Key learning points}

$>$ Objective weakness may be subtle in early autoimmune myositis

$>$ Close examination of the hands - 'the calling card of rheumatology' is important in patients presenting with possible myositis $^{6}$

> Mildly raised muscle-enzyme levels can be an important pointer to significant muscle disease

$>$ MRI is a very useful non-invasive tool in the investigation of myositis

MRI = magnetic resonance imaging.

muscle fibres, but this test is invasive and requires specialised histology expertise. ${ }^{3} \mathrm{MRI}$ is sensitive but not specific for autoimmune myositis. In certain clinical contexts, such as in this case, MRI can help to confirm the diagnosis. ${ }^{5}$ It can also be useful in identifying potential biopsy sites. ${ }^{5}$

Dermatomyositis is associated with an increased risk of malignancy, and current recommendations are to investigate all those over 40 years who have dermatomyositis for breast, ovarian, lung and gastrointestinal-tract tumours specifically. ${ }^{2}$ Because of this increased risk, a comprehensive history and physical examination is important. ${ }^{1}$ Breast and pelvic examinations are recommended in women and testicular and prostate examinations in men. ${ }^{3}$ A CT chest, abdomen and pelvis is recommended with the addition of a pelvic ultrasound and mammogram for women. ${ }^{3}$ Colonoscopy is advisable for all those aged over 50 years. $^{3}$

Treatment for dermatomyositis consists of conservative measures including the use of sun-blocking agents and physical activity to maintain strength. ${ }^{1}$ Corticosteroids are the first-line treatment. ${ }^{3}$ Second-line agents include methotrexate, azathioprine, mycophenolate mofetil and intravenous immunoglobulins. ${ }^{1}$

\section{References}

1 Mehta G, Iqbal B. Clinical medicine for the MRCP PACES: Volume 1 core clinical skills. Oxford: Oxford University Press, 2010.

2 Ryder REJ, Afzal M, Freeman A, Fogden E. An Aid to the MRCP PACES: Volume 3: Station 5, 4th edn. Oxford: Wiley-Blackman, 2013.

3 Amato AA, Greenberg SA. Inflammatory myopathies. Continuum (Minneap Minn) 2013;19(6 Muscle Disease):1615-33.

4 Miller M, Vleugels RA. Clinical manifestations of dermatomyositis and polymyositis in adults. UpToDate, 2013; www.uptodate.com/ contents/clinical-manifestations-of-dermatomyositis-andpolymyositis-in-adults [Accessed 31 July 2014].

5 Carstens PO, Schmidt J. Diagnosis, pathogenesis and treatment of myositis: recent advances. Clin Exp Immunol 2014;175:349-58.

6 Da Silva JAP, Woolf AD. Rheumatology in practice. London: Springer, 2010.

Address for correspondence: Dr A Ratnarajah, Acute Medicine, Frimley Park Hospital, Portsmouth Road Frimley Surrey GU16 7UJ.

Email: abarna.ratnarajah@fph-tr.nhs.uk 